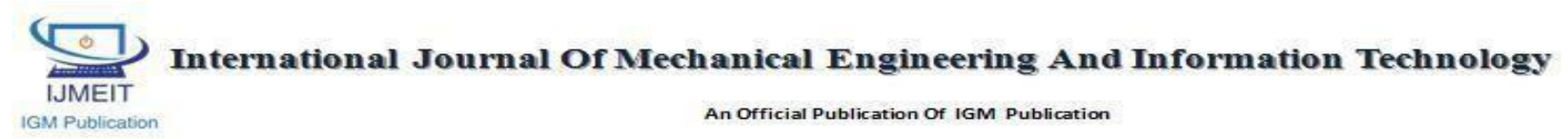

\title{
A Survey on SAR image Georegistration and Orthorectification
}

Authors

\author{
Nagashree. R.N ${ }^{1}$, Vibha Rao ${ }^{2}$ \\ ${ }^{1}$ Asst.Prof, Telecommunication Engineering, GSSSIETW, Mysuru, \\ ${ }^{2}$ Asst.Prof, Electronics \& Communication Engineering, NIEIT, Mysuru, \\ Email-nagashree.r.n@gmail.com,vibha.rao89@gmail.com
}

\begin{abstract}
:
This paper outlines the principle of Synthetic Aperture Radar (SAR). Also In this paper we have discussed what the factors are that results in SAR image distortion. A brief introduction to Geo Registration and Ortho rectification can be seen here. The distortion resulted in SAR image can be corrected by Geo Registration. Registration algorithms compute transformations to set correspondence between the two images. Hence a Registration process is a aligning of two images into a common coordinate system and then aligning them in order to monitor subtle changes between the two. But not all the distortion can be removed that includes distortions brought by elevation and it is very difficult to correct in Geo Registration method. Hence we go for Orthorectification which removes the perspective and terrain relief in the image for the purpose of creating a planimetrically correct image. This paper discusses about the problems occurring in SAR radar. Keyword: Digital Elevation Model (DEM), Ground Control points (GCP), Synthetic Aperture Radar (SAR), Referenced image, Sensed Image.
\end{abstract}

\section{INTRODUCTION}

The Synthetic Aperture Radar has a long history in developing technologies and components since from 50 years. Since then a rapid development is taking all over the world. SAR is an air and spaceborne systems which are operational till today. Environmental monitoring, earth-resource mapping, and military systems require broad-area imaging at high resolutions. SAR has proved valuable because of its day and night capability and the possibility to penetrate clouds and rain. The ability of SAR to calculate azimuth range and to produce relatively fine azimuth resolution differentiates it from other radars. SAR systems take advantage of the long-range propagation characteristics of radar signals and the complex information processing capability of modern digital electronics to provide high resolution imagery. Synthetic aperture radar complements photographic and other optical imaging capabilities because of the minimum constraints on time-of-day and atmospheric conditions and because of the unique responses of terrain and cultural targets to radar frequencies.

Synthetic aperture radar technology has provided terrain structural information to geologists for mineral exploration, oil spill boundaries on water to environmentalists, sea state and ice hazard maps to navigators, and reconnaissance and targeting information to military operations. There are many other applications such as remote sensing and mapping of the surfaces of both the Earth and other planets.

The idea of SAR was to transmit pulses and store the scene echoes along a synthetic aperture (i.e. the path of the SAR sensor) and to combine the echoes afterwards by the application of an appropriate focusing algorithm. The combination is carried out coherently.

The synthetic aperture results from the movement of the source as it emits the pulses. The two images which we get from different sensors, different time and different view point can have different intensity values and there will be no general mapping between those images. But many edge contours, region features, line features 
human made objects and other boundaries will be similar between the two types of images. Extracting strong edges from SAR images is difficult task due to large amount of speckles in SAR images. Speckle is a multiplicative noise combined with the image due to the process of coherent imaging.

To compare the multiple images which were resulted due to the above mention facts results in Georegistration of images. The definition and process of Georegistration goes likethis.

Georegistration is the alignment of an unreferenced image with a geodetically (latitude, longitude, and elevation based) calibrated reference image. In general, this process aligns two images (usually satellite images) and correlates the images to a physical location by examining a set of distinguishable points.

The Georegistration process then requires the image to be transformed so that all matching points can be matched. This alignment and correlation makes it possible to compare multiple images from different sensors or times that have different viewing perspectives due to changes in location, angle, refraction, sensor differences, resolution differences, and many other image distortions. The major difference between the Image registration and Georegistration is, Georegistration works with geographically based reference points while Image-registration focus on a variety of less- specifiable points, depending on the application.

Hence to detect the changes in satellite images, accurate geo-referencing information is a basic requirement and it is extremely important for the images to be precisely Georegistered and orthorectified.

Images from high-resolution satellites are now relatively widely used for a variety of applications. For use in medium and large-scale mapping applications, it is desirable that image to be orthorectified. It determines the image resolution and horizontal accuracy. Orthorectification implies that both sensor system and terrain related errors are reduced to some specified level and that the image pixels are resampled to a particular projection (i.e., from the perspective projection to orthogonal projection) and reference system. Traditional Orthorectification involves the use of stereo pairs from which elevation is extracted directly to be used for contours and Digital Elevation Models (DEMs) in addition to Orthorectification of the images ${ }^{[1,2,6,8,9,12,13]}$.

In detail description of principle of SAR, Georegistration, Orthorectification are given in the following next topics.

\section{PRINCIPLE OFSAR}

A Synthetic Aperture Radar is an airborne or space borne Side-Looking Radar Systems which utilizes the flight path of the platform and generates high resolution remote sensing images. Hence in SAR extremely large antenna are used. The SAR works similar of a phased array, but contrary of a large number of the parallel antenna elements of a phased array, SAR uses one antenna in time-multiplex. The different geometric positions of the antenna elements are result of the moving platform now. Range measurement and resolution are achieved in SAR is as same as obtaining in other radars. Range is determined by measuring the time from transmission of a pulse to receiving the echo from a target. The SAR processor stores all the radar returned signals as amplitudes and phases. Now it is possible to reconstruct the signal. As the line of sight direction changes along the radar platform trajectory, a synthetic aperture is produced by signal processing that has the effect of lengthening the antenna hence a higher resolution can be achieved. The typical operation of a space borne SAR is as follows.

Each pulse is transmitted to the target area where the antenna beam intercepts the earth and illuminates the target at that location and the reflected return pulses from the target are in turn collected by the same antenna as it is a duplexer. The SAR system saves the phase histories of the responses at each position as the real beam moves from the scene and then weights, phase shifts, and 
sum them to focus on one point target at a time and suppress all others. Next SAR image signal processing performs the above techniques to focus on each point target inturn.

It then constructs an image by placing total energy response obtained in the focusing on a particular target at the position in the image corresponding to that target.

Airborne radar could collect data while flying this distance and then process the data as if it came from a physically long antenna. The distance the aircraft flies in synthesizing the antenna is known as the synthetic aperture. A narrow synthetic beam width results from the relatively long synthetic aperture, which yields finer resolution than is possible from a smaller physical antenna. Achieving fine azimuth resolution may also be described from a Doppler processing viewpoint. A target's position along the flight path determines the Doppler frequency of its echoes: Targets ahead of the aircraft produce a positive Doppler offset; targets behind the aircraft produce a negative offset. As the aircraft flies a distance (the synthetic aperture), echoes are resolved into a number of Doppler frequencies. The target's Doppler frequency determines its azimuth position $[1,2,9$, and 10$]$

\section{A.ERS SAR geometric configuration}

The spacecraft is flying in its orbit and carries a SAR sensor which points perpendicular to the flight direction. The projection of the orbit down to Earth is known as the ground track or sub satellite track. The area continuously imaged from the radar be a miscalled radar swath. Due to the look angle of about 23 degrees in the case of ERS, the imaged area is located about $250 \mathrm{~km}$ to the right of the sub satellite track. The radar swath itself is divided in a near range - the part closer to the ground track - and a farrange.

In the SAR image, the direction of the satellite's movement is called azimuth direction, while the imaging direction is called range direction ${ }^{[10]}$.

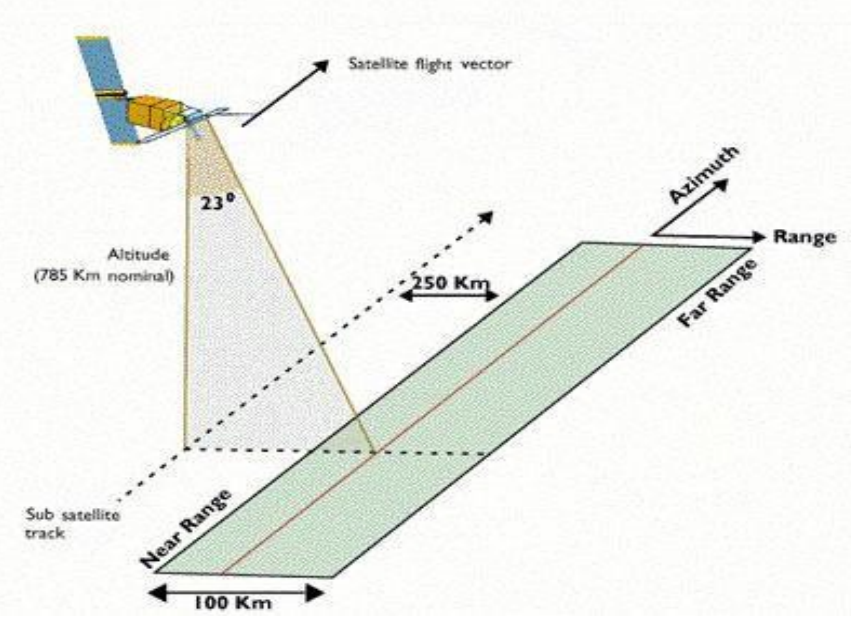

Fig. 1. Shows the different ranges of SAR ${ }^{[10]}$ The design of modern SAR Systems demands for increasing resolution capabilities. With the longer antennas, angle of the beam is increased this gives the increase in antenna gain and operational ranges. Also steerable antennas can be used so that there will be an illumination of larger aspect of angles. Hence longer antennas are preferred over a shorter antenna in SAR.

\section{PROBLEMS FACED INSAR}

The distortions inherent in the images of the satellite are due to

\section{A. The topographical variations in the surface of the earth}

The topographical variations of the earth's surface and the satellite view zenith angle affect the pixel's distance projected onto the satellite image. The distortion inherent in the image is determined by topographical elevation. For example, an offnadir view of 5 degrees at a site with a terrain height of 1000 meters can cause displacement of 120 meters (about 4 Landsat Thematic Mapper (TM) pixels). The Orthorectification process is used to correct the pixel displacement caused by the topographical variations at the off-nadir viewing and to make the image orthographic, with every pixel in its correct location regardless of elevation and viewing direction.

B. Geometrical deformations: The geometrical deformations results from the way of sorting points by using their distance to the antenna. The ground position of a point can be slightly (or 
more) wrong because it has been "seen" as shifted toward the antenna.

- Slant Range Distortion: The slant-range distortion occurs because the radar is measuring the distance to features in slantrange rather than the true horizontal distance along the ground. This results in a varying image scale, moving from near to farrange.

- Range Distortion: Radar measures slant ranges but, for an image to represent correctly the surface, it must be ground range corrected.

- Elevation Distortion: This occurs in those cases where points have an elevation different from the mean terrain elevation.

- Shadow Effects: A slope away from the radar illumination with an angle that is steeper than the sensor depression angle provokes radar shadows. For example let us have a hill as an object, when the SAR senses the object it takes a slant range of measurement hence only one part of the hill is detected while the other is not detected. The part which is not detected is usually known as radar shadow. This is shown by the dark zones in the central part of the image.

- Foreshortening: This occurs when the radar beam reaches the base of a tall feature tilted towards the radar (e.g. a mountain) before it reaches the top. Because the radar measures distance in slant-range, the slope will appear compressed and the length of the slope will be represented in correctly at the image plane. If mountain is taken as a then the same value a will not be received its feature will be changed to a'.

- Layover: This occurs when the radar beam reaches the top of a tall feature before it reaches the base. The return signal from the top of the feature will be received before the signal from the bottom. As a result, the top of the feature is displaced towards the radar from its true position on the ground, and layover the base of the feature. If a mountain is selected from top to bottom as $\mathrm{a}$ and $\mathrm{b}$ then after the effects layover it will be a' and $b^{,}[1,2,11]$.

\section{IMAGEGEOREGISTRATION}

The Georegistration process geometrically aligns two images with different viewing geometry and/or different terrain distortions into the same coordinate system so that corresponding pixels represent the same objects. The process includes locating and matching a number of feature points (called tie points) in two images (a warp image and a base image) selected for registration. The corresponding tie points are used to compute the parameters of a geometric transformation between the two images.

The need to register images came from many practical problems including the integration of information from multiple sensors, finding changes in images across times and differing conditions, inferring three-dimensional information from images in which either the camera or the objects moved, and object recognition within a model. Georegistration was simply an extension of the image-registration to include a geographically based reference image. To have the accuracy Global Positioning System were included into the calculations of Georegistration.

This change provided a means for increasing the efficiency and reliability of measurements taken from multiple sensors. This addition of GPS has proved to be one of the greatest advances since the 1980s

The Georegistration process then requires the image to be transformed so that all matching points can be matched. This alignment and correlation makes it possible to compare multiple images from different sensors or times that have different viewing perspectives due to changes in location, angle, refraction, sensor differences, resolution differences, and many other image distortions.

Common Steps involved in Georegistering an 
image

A. Feature Detection: To match the reference image with the sensed image we need to detect the objects that are common to both which are called as control points. In Feature detection is the method we use intensity or line features, region features, point features to calibrate the control points. This is done using two primary methods. In the first method Digital Elevation Map is used to calibrate the control points but in this method there will be some random noise and other distortions. Hence an alternative and more commonly used second method to detect control points is based on image features. It depends upon intensity based algorithm rather than Elevation.

B. Feature matching: After the detection of control points it should be mapped between reference image and sensed image.

C. Transformation Model: Once the control points have been identified and matched between the images, depending upon the set of pair of control points, the transformation that is needed to be done is decided. This functional transformation is called as Georectification of an image. The same transformation can be applied to the whole image or for each image in an entire sensed image. These types of transformation are called as Global Mapping (former) and Local Mapping (Lateral).

\section{Image Transformation and Resampling: The}

final step is to transform the image based on the model created by the previous steps. . These transformations of an image are also known as "rubber-sheeting" or "rubber-masking". The concept of "rubber-sheeting" is the process of reprojecting an image using methods to interpolate pixels in order to adjust the image to a greater or lesser number of pixels. This is not done to adjust the resolution of the image, but rather to make adjustments for angular shifts or translations that do not allow the images to accurately correspond to each other. The particular transformations in the "rubber-sheeting" are applied between control points rather than within the image as a whole. Often, different interpolation algorithms are applied to result in the most graceful translation between the original and the corrected image. Once this translation has occurred, the image has been aligned at each control point. The result of "rubber-sheeting" goes a step further than the traditional transformations to include the ability to transform some parts of an image to a greater extent than others. This may be needed when an image has been composited or if a sensor is not completely consistent through the whole viewing area. One remaining point which requires attention is the step of registering an initial image to a physical location, through latitude and longitude. The process to create the control image is not trivial due to selecting identifiable control points as well as identifying the geodetic data for that control image. The Georegistration is an iterative process ${ }^{[3,4,5,11]}$.

\section{IMAGEORTHORECTIFICATION}

Orthorectification is the process of removing the effects of image perspective (tilt) and relief (terrain) effects for the purpose of creating a planimetrically correct image. An Orthorectified image gives the accurate direct measurement of distances, angles, and areas (i.e. mensuration). Orthorectified images are commonly used as in visualization tools such as Google Earth, OSSIM Planet, Arc Map, WMS, etc.

The distortions in a sensed image caused by the relief cannot in general be corrected by Georegistration, even with a transformation of high degree (say, 3rd degree polynomial). When the relief is moderate the target image should be corrected using a digital elevation model. This can be done as long as one knows what is the precise geometry of acquisition of the image (aerial photography, satellite imagery). Orthorectification is done in two steps. Firstly, the (column, row) coordinates of the target image pixels are converted into 3D (x; y; z) coordinates.

Secondly, the $(\mathrm{x} ; \mathrm{y} ; \mathrm{z})$ coordinates are projected onto the surface. This can be interpreted as conversion of perspective image to orthogonal image. This can be explained in more detail. 
The topographical variations in the surface of the earth and the tilt of the satellite or aerial sensor affect the distance with which features on the satellite or aerial image are displayed. If the landscapes are topographically more diversified then we can see more distortion in the photograph. If the sensor is not pointing directly at the nadir location of the sensor then there will be introduction of terrain distortions and geometrical errors. Orthorectification process will be used here. It transforms the central projection of the photograph (perspective projection) into an orthogonal view of the ground (orthogonal projection), thereby removing the distorting effects of tilt and terrain relief. Without Orthorectification scale is not constant in the image and accurate measurements of distance and direction cannot be made.

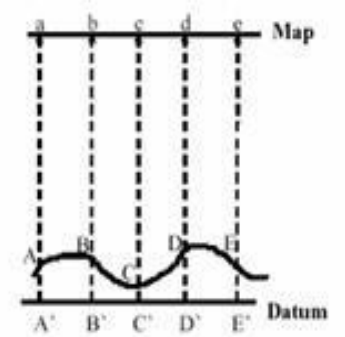

- Orthogonal projection.

- Uniform scale.

- No relief displacement.

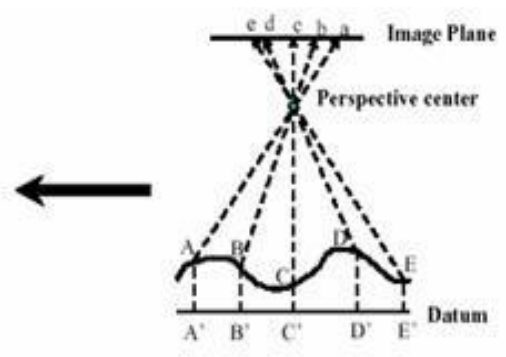

- Perspective projection.

- Non-uniform scale.

- Relief displacement.
Fig. 2. Orthogonal vs. perspective projections ${ }^{[12]}$ From the above discussion we can say that orthoimage are plan imetrically correct and it can be an effective tool for use in resource management, municipal planning, cadastral mapping, and geographic information systems (GIS).

Even after Orthorectification process some of the objects like buildings, bridges, trees etc., remain in perspective views and are distorted from their true position. Distortions show as, for instance, leaning buildings and bent bridges. Some interesting information from ground features like streets and other objects are hidden from the user of the orthoimage. Furthermore the superimposition of vector data is nearly impossible. If Digital Surface Models (DSMs) describing the mentioned objects that caused the displacement are used for the Orthorectification processes, the displacements can be corrected and the results are called "True Orthoimage".

An Orthorectification algorithm includes polynomial transformation and projective transformation. Still if there is any height displacement errors then complex algorithms can be included ${ }^{[6,7,11,12,13]}$.

\section{ORTHORECTIFICATION VS GEOREGISTRATION}

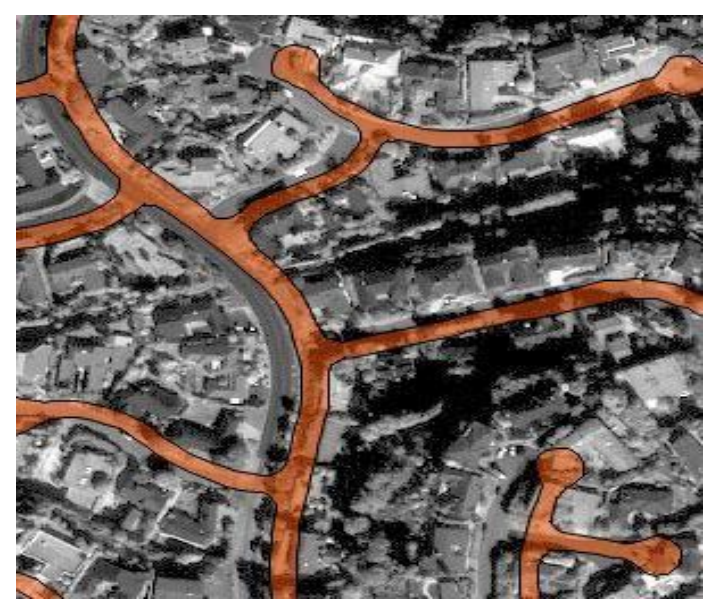

Fig. 3. This image is Georegistered but not Orthorectified [courtesy ISA]

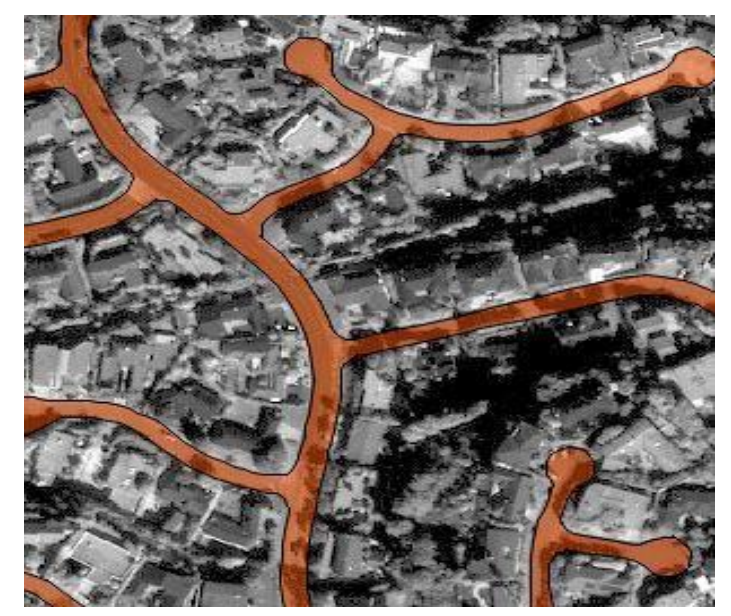

Fig. 4. This image is both Georegistered and Orthorectified [courtesy ISA]

\section{CONCLUSION}

This paper describes the SAR mainly as a Remote Sensing Radar. And it discusses about the basic principle of SAR and distortions created during taking the aerial photo or satellite photo. Only the Georegistration is not enough to remove the 
distortion even though Digital Elevation Models are used. To have the accurate measurement of the sensed photo (i.e., which we are getting from the target) Orthorectification is needed. Hence introduction of Georegistration and Orthorectification are given in this paper. Also not all the Georegistered images are orthorectified but we can have the liberty to say all the Orthorectified images are Georegistered.

\section{REFERENCES}

1. Y. K. Chan and V. C. Koo, "An Introduction to Synthetic Aperture Radar (SAR)," Progress in Electromagnetics Research B, vol. 2, 27-60,2008.

2. Dr. Patrick Berens, "Introduction to Synthetic Aperture Radar (SAR)," Advanced Radar Signal and Data Processing pp. 3-1 -3- 14.

3. Suvesh Kumar, K. V. Arya, Vinay Rishiwal, P. N. Joglekar, "Robust Image registration technique for SAR images," First International Conferenceon Industrial and Information Systems, ICIIS 2006,811August2006,SriLanka.

4. Pamann, S. Mavromatics, J. Sequeria, "SAR Image registration using a new approach based on the generalized Hough Transform," The International Archives of the Photogrammetry, Remote Sensing and Spatial Information Sciences, vol. XXXVII, Part B7, Beijing2008.

5. Woo-Kyung Lee, Ah-Leum Kim, "An efficient Automatic Geo-Registration Technique for high resolution spaceborne SAR Image Fusion," IEEE,2011.

6. J. Bryan Mercer, Jermey Allan, Natallic Glass, Jonathon Rasmussen, Michel Wollersheim, "Orthorectification of satellite images using external DEMS from IFSAR,'ComissionII,WorkingGroupII/2,I ntermapTechnologiesCorp.

7. Leland Pierce,Josef Kellndorfer, Fawwaz Ulbay, "Practical SAR Orthorectification,"IEEE1996.
8. Chrisoliver, Shaun Quegan, "Understanding Synthetic Aperture Radar Images".

9. SamuelW.(Walt)McCandless,Jr,Christoph erR.Jackson,"Principles of Synthetic Apertue Radar".

10. Dr. S. C. Liew, "Principles of Remote Sensing".

11. Didier Massonnet, Jean-Claude Sourvis, "Imaging with Synthetic Aperture Radar".

12. Dr. F. I. Okeke, "Review of Digital Image Orthorectification Techniques".

13. Satellite Imaging Corporation, "www.satimagingcorp.com". 